\title{
QUANTUM SUPERPOSITION PRINCIPLE JUSTIFIED IN A NEW NON-ARISTOTELIAN FINITARY LOGIC*
}

\author{
Radhakrishnan Srinivasan ${ }^{\dagger}$
}

\begin{abstract}
In the proposed non-Aristotelian finitary logic (NAFL), truths for formal propositions can exist only with respect to axiomatic theories, essentially as temporary axiomatic declarations in the human mind. An undecidable proposition $P$ in a consistent NAFL theory $\mathrm{T}$ is true/false with respect to $\mathrm{T}$ if and only if it has been axiomatically declared as true/false by virtue of its provability/refutability in an interpretation $\mathrm{T}^{*}$ of $\mathrm{T}$. In the absence of any such axiomatic declarations, $P$ is in a superposed state of 'neither true nor false' and consistency of $\mathrm{T}$ requires the existence of a non-classical model for $\mathrm{T}$ in which $P \& \neg P$ is the case. Here $\mathrm{T}^{*}$ is an axiomatic NAFL theory that, like $\mathrm{T}$, resides in the human mind and acts as the 'truth-maker' for (a model of) T. Quantum superposition is justified by identifying 'axiomatic declarations' for the truth/falsity of $P$ (by virtue of its provability/refutability in $\mathrm{T}^{*}$ ) with 'measurement' in the real world. NAFL also explains and de-mystifies the phenomenon of entanglement. NAFL severely restricts classical infinitary reasoning, but possibly provides sufficient machinery for a consistent axiomatization of quantum mechanics.
\end{abstract}

Keywords: quantum superposition, entanglement, non-classical logic, finitary reasoning.

*IBM is a trademark of the International Business Machines Corporation in the Unites States, other countries, or both.

${ }^{\dagger} \mathrm{R} \&$ \& Group, IBM India Software Labs, 5th floor, Golden Enclave, Airport Road, Bangalore 560017, India. E-Mail: sradhakr@in.ibm.com 


\section{Introduction to Non-Aristotelian Finitary Logic (NAFL)}

The language, well-formed formulae and rules of inference of NAFL theories $[1,2]$ are formulated in exactly the same manner as in classical first-order predicate logic with equality (FOPL), where we shall assume, for convenience, that natural deduction is used; however, there are key differences and restrictions imposed by the requirements of the Main Postulate of NAFL, which is explained in this section. In NAFL, truths for formal propositions can exist only with respect to axiomatic theories. There are no absolute truths in just the language of an NAFL theory, unlike classical/intuitionistic/ constructive logics. There do exist absolute (metamathematical, Platonic) truths in NAFL, but these are truths about axiomatic theories and their models. As in FOPL, an NAFL theory $\mathrm{T}$ is defined to be consistent if and only if $\mathrm{T}$ has a model, and a proposition $P$ is undecidable in $\mathrm{T}$ if and only if neither $P$ nor its negation $\neg P$ is provable in $\mathrm{T}$.

\section{The Main Postulate of NAFL}

If a proposition $P$ is provable/refutable in a consistent NAFL theory $\mathrm{T}$, then $P$ is true/false with respect to $\mathrm{T}$ (henceforth abbreviated as 'true/false in T'); i.e., a model for $\mathrm{T}$ will assign $P$ to be true/false. If $P$ is undecidable in a consistent NAFL theory T, then the Main Postulate [2] provides the appropriate truth definition as follows: $P$ is true/false in $\mathrm{T}$ if and only if $P$ is provable/refutable in an interpretation $\mathrm{T}^{*}$ of $\mathrm{T}$. Here $\mathrm{T}^{*}$ is an axiomatic NAFL theory that, like $\mathrm{T}$, temporarily resides in the human mind and acts as a 'truth-maker' for (a model of) $\mathrm{T}$. The theorems of $\mathrm{T}^{*}$ are precisely those propositions that are assigned 'true' in the NAFL model of T, which, unlike its classical counterpart, is not 'pre-existing' and is instantaneously generated by $\mathrm{T}^{*}$. For a given consistent theory $\mathrm{T}, \mathrm{T}^{*}$ could vary in time according to the free will of the human mind that interprets $\mathrm{T}$. Hence $\mathrm{T}^{*}$ could be $\mathrm{T}+P$ on a given day, $\mathrm{T}+\neg P$ on another, or just $\mathrm{T}$ itself on a third day, where $P$ is undecidable in $\mathrm{T}$; the essence of the Main Postulate is that $P$ is true/false in $\mathrm{T}$ if and only if it has been axiomatically declared as true/false by virtue of its provability/refutability in $\mathrm{T}^{*}$. In the absence of any such axiomatic declarations, i.e., if $P$ is undecidable in $\mathrm{T}^{*}\left(e . g\right.$. take $\left.\mathrm{T}^{*}=\mathrm{T}\right)$, then $P$ is 'neither true nor false' in $\mathrm{T}$ and Proposition 1 shows that consistency of $\mathrm{T}$ 
requires the laws of the excluded middle and non-contradiction to fail in a non-classical model for $\mathrm{T}$ in which $P \& \neg P$ is the case.

Proposition 1. Let $P$ be undecidable in a consistent NAFL theory T. Then $P \vee \neg P$ and $\neg(P \& \neg P)$ are not theorems of $\mathrm{T}$. There must exist a nonclassical model $\mathcal{M}$ for $\mathrm{T}$ in which $P \& \neg P$ is the case.

The interpretation of $P \& \neg P$ in the non-classical model will be explained in Sec. 2. Proposition 1 is a metatheorem, i.e., it is a theorem about axiomatic theories. The concepts in Proposition 1, namely, consistency, undecidability (provability) and the existence of a non-classical model for a theory (and hence, quantum superposition and entanglement), are strictly metamathematical and not formalizable in NAFL theories. An NAFL theory T is either consistent or inconsistent, and a proposition $P$ is either provable or refutable or undecidable in $\mathrm{T}$, i.e., the law of the excluded middle applies to these metamathematical truths.

Proof. By the Main Postulate of NAFL, $P(\neg P)$ can be the case in $\mathrm{T}$ if and only if $P(\neg P)$ has been asserted axiomatically, by virtue of its provability in $\mathrm{T}^{*}$. In the absence of any such axiomatic assertions $\left(e . g\right.$. if $\left.\mathrm{T}^{*}=\mathrm{T}\right)$, it follows that neither $P$ nor $\neg P$ can be the case in $\mathrm{T}$ and hence $P \vee \neg P$ cannot be a theorem of T. The classical refutation of $P \& \neg P$ in T proceeds as follows: 'If $P(\neg P)$ is the case, then $\neg P(P)$ cannot be the case', or equivalently, ' $\neg P(P)$ contradicts $P(\neg P)^{\prime}$ '. But, by the Main Postulate, this argument fails in NAFL and amounts to a refutation of $P \& \neg P$ in $\mathrm{T}^{*}=\mathrm{T}+P(\mathrm{~T}+\neg P)$, and not in $\mathrm{T}$ as required. Careful thought will show that the classical refutation of $P \& \neg P$ in $\mathrm{T}$ is the only possible reason for $\neg(P \& \neg P)$ to be a theorem of $\mathrm{T}$, and it fails in NAFL. The intuitionistic refutation of $P \& \neg P$ in $\mathrm{T}$ is flawed and also fails in NAFL, as will be shown below. By the completeness theorem of FOPL, which is taken for granted in NAFL, it follows that there must exist a non-classical model for $\mathrm{T}$ in which $P \& \neg P$ is satisfiable.

Consider the law of non-contradiction as stated in a standard system of intuitionistic first-order predicate logic due to S. C. Kleene, namely, $\neg P \Rightarrow(P \Rightarrow Q)$. This formula asserts that from contradictory premises $P$ and $\neg P$, an arbitrary proposition $Q$ can be deduced, which is absurd. Hence $\neg(P \& \neg P)$ seemingly follows. However, note that in intuitionism, truth is provability (not necessarily in a specific theory $\mathrm{T}$ ); together with the intuitionistic concept of negation, it follows that an asssertion of $\neg(P \& \neg P)$ 
is the same as deducing an absurdity from $P \& \neg P$, or equivalently, from contradictory premises $P$ and $\neg P$. But we have seen that the 'absurdity' referred to here is precisely the fact that any proposition can be deduced, given contradictory premises! The above 'proof' of $\neg(P \& \neg P)$ from contradictory premises, mandated by the intuitionistic concepts of truth and negation, is flawed because any proposition can be so deduced. Note that this 'proof' is formally indistinguishable from one in which $\neg(P \& \neg P)$ is substituted for the arbitrary proposition $Q$. In NAFL, it is not possible to deduce an arbitrary proposition from contradictory premises [1] in a non-classical model, and so the flawed intuitionistic argument for $\neg(P \& \neg P)$ fails in any case. Indeed, as explained in Ref. [1], the argument for deducing an arbitrary proposition would normally proceed as follows:

$$
\begin{aligned}
& P \& \neg P \Rightarrow P, \\
& P \Rightarrow P \vee Q, \\
& P \& \neg P \Rightarrow \neg P, \\
& \neg P \&[P \vee Q] \Rightarrow Q .
\end{aligned}
$$

The final step fails in a non-classical NAFL model for a theory $\mathrm{T}$ (in which $P \& \neg P$ is the case) because this step presumes the law of non-contradiction for $P$. Note that the existence of this non-classical model does not make $\mathrm{T}$ inconsistent or paraconsistent, because $\mathrm{T}$ does not prove $P \& \neg P$. However, one could assert that model theory for NAFL requires the framework of a paraconsistent logic, so that the non-classical models can be analyzed. NAFL is the only logic that correctly embodies the philosophy of formalism [2]; NAFL truths for formal propositions are axiomatic, mental constructs with strictly no Platonic world required.

An NAFL theory $T$ requires two levels of syntax, namely the 'theory syntax' and the 'proof syntax'. The theory syntax consists of precisely those propositions that are legitimate, i.e., whose truth in $\mathrm{T}$ satisfies the Main Postulate; obviously, the axioms and theorems of $\mathrm{T}$ are required to be in the theory syntax. Further, one can only add as axioms to $\mathrm{T}$ those propositions that are in its theory syntax. In particular, neither $P \& \neg P$ nor its negation $P \vee \neg P$ is in the theory syntax when $P$ is undecidable in T. The proof syntax, however, is classical because NAFL has the same rules of inference as FOPL; thus $\neg(P \& \neg P)$ is a valid deduction in the proof syntax and may be used to prove theorems of T. For example, if one is able to deduce $A \Rightarrow P \& \neg P$ in the proof syntax of T where $P$ is undecidable in $\mathrm{T}$ and $A$ is in the theory syntax, 
then one has proved $\neg A$ in $\mathrm{T}$ despite the fact that $\neg(P \& \neg P)$ is not a theorem (in fact not even a legitimate proposition) of $\mathrm{T}$. This is justified as follows: $\neg(P \& \neg P)$ may be needed to prove theorems of $\mathrm{T}$, but it does not follow in NAFL that the theorems of T imply $\neg(P \& \neg P)$ if $P$ is undecidable in T. Let $A$ and $B$ be undecidable propositions in the theory syntax of T. Then $A \Rightarrow B$ (equivalently, $\neg A \vee B$ ) is in the theory syntax of $\mathrm{T}$ if and only if $A \Rightarrow B$ is not (classically) deducible in the proof syntax of $\mathrm{T}$. It is easy to check that if $A \Rightarrow B$ is deducible in the proof syntax of $\mathrm{T}$, then its (illegal) presence in the theory syntax would force it to be a theorem of $\mathrm{T}$, which is not permitted by the Main Postulate: in a non-classical model for $\mathrm{T}$ in which both $A$ and $B$ are in the superposed state, $A \& \neg B$ must be non-classically true. If one replaces $B$ by $A$ in this result, one obtains the previous conclusion that $\neg(A \& \neg A)$ is not in the theory syntax. For example, take $\mathrm{T}_{0}$ to be the null set of axioms. Then nothing is provable in $\mathrm{T}_{0}$, i.e., every legitimate proposition of $\mathrm{T}_{0}$ is undecidable in $\mathrm{T}_{0}$. In particular, the proposition $(A \&(A \Rightarrow B)) \Rightarrow B$, which is deducible in the proof syntax of $\mathrm{T}_{0}$ (via the modus ponens inference rule), is not in the theory syntax; however, if $A \Rightarrow B$ is not deducible in the proof syntax of $\mathrm{T}_{0}$, then it is in the theory syntax. Note also that $\neg \neg A \Leftrightarrow A$ is not in the theory syntax of $\mathrm{T}_{0}$; nevertheless, the 'equivalence' between $\neg \neg A$ and $A$ holds [2] in the sense that one can be replaced by the other in every model of $\mathrm{T}_{0}$, and hence in all NAFL theories. Indeed, in a non-classical model for $\mathrm{T}_{0}$, this equivalence holds in a non-classical sense and must be expressed by a different notation [2].

NAFL restricts classical infinitary reasoning [3] - infinite sets cannot exist in consistent NAFL theories (see Sec. 3 of Ref. [1]). However, if an NAFL theory $\mathrm{T}$ admits infinitely many objects satisfying a given property, then $\mathrm{T}$ must necessarily prove the existence of the corresponding infinite (proper) class $[2,3]$. Cantor's diagonalization argument fails because it requires quantification over infinitely many proper classes (e.g., real numbers), which is banned in NAFL. For this reason, Gödel's incompleteness theorems do not apply to, and Turing's halting problem must be decidable in, NAFL theories [2]. A Turing machine (TM), by definition, must either halt or not halt; the superposed state of the TM (in which it neither halts nor not halts), required by Proposition 1 for undecidability of halting, is not possible in NAFL. Indeed, such a superposed state would immediately imply that the TM in fact does not halt. In effect, NAFL bans the self-reference in Cantor's and Turing's arguments. Non-standard models of arithmetic (and hence, infinite integers or infinitesimals) cannot exist in NAFL [2]; it follows that 
consistency of Peano Arithmetic demands its completeness. Future work will outline how a limited version of real analysis can be executed in NAFL, despite these restrictions. This should pave the way for a consistent formalization of quantum mechanics in a single logic (NAFL), without the present need to abruptly jump from quantum logic to FOPL.

\section{Quantum Superposition and Entanglement Justified in NAFL}

The non-classical model $\mathcal{M}$ of Proposition 1 is a superposition of two or more classical models for $\mathrm{T}$, at least in one of which $P$ is true and $\neg P$ in another. Here '(non-)classical' is used strictly with respect to the status of $P$. In $\mathcal{M}$, ' $P$ ' (' $\left.\neg P^{\prime}\right)$ denotes that ' $\neg P$ ' (' $P$ ') is not provable in $\mathrm{T}^{*}$, or in other words, $\mathcal{M}$ expresses that neither $P$ nor $\neg P$ has been axiomatically declared as (classically) true in T; thus $P, \neg P$, and hence $P \& \neg P$, are indeed (non-classically) true in our world, according to this interpretation. Note also that $P$ and $\neg P$ are classically 'neither true nor false' in $\mathcal{M}$, where 'true' and 'false' have the meanings given in the Main Postulate. The quantum superposition principle is justified by identifying 'axiomatic declarations' of truth/falsity of $P$ in $\mathrm{T}$ (via its provability/refutability in $\mathrm{T}^{*}$ as defined in the Main Postulate) with 'measurement' in the real world. NAFL is more in tune with the Copenhagen interpretation of quantum mechanics than the many-worlds interpretation (MWI). Nevertheless, the information content in $\mathcal{M}$ is that of two or more classical models (or 'worlds'), and MWI is at least partially vindicated in this sense. Consider the Schrödinger cat example, where the cat is put into the box at time $t=0$ and has a probability 0.5 of being in the 'alive' state at $t=1$, when a 'measurement' is made of its state. Let $P$ be the proposition that 'The cat is alive', with $\neg P$ denoting 'The cat is dead'; obviously, $P$ is undecidable in a suitable formalization QM of quantum mechanics. For $0<t<1$, the observer makes no measurements, and in tune with the above identification, makes no axiomatic declarations regarding $P$ in the interpretation $\mathrm{QM}^{*}$ (say, let $\mathrm{QM}^{*}=\mathrm{QM}$ for this time period). In the resulting non-classical model $\mathcal{M}$ of $\mathrm{QM}$, the superposed state $P \& \neg P$ is the

case; this means that the cat has not been declared (measured) to be either alive or dead, which is certainly true in the real world. At $t=1$, if $P(\neg P)$ is observed, then the observer takes, say, $\mathrm{QM}^{*}=\mathrm{QM}+P(\neg P)$; i.e., when 
the observer measures the cat to be alive (dead) in the real world, he makes the appropriate axiomatic declarations in his mind, thus setting up QM* as defined. It should be emphasized that an NAFL theory only 'sees' the observer's axiomatic declarations and does not care whether the real world exists. The observer sees the real world and the proposed identification of his measurements with his axiomatic declarations is only an informal convention that is outside the purview of NAFL. The observer could also use his free will to make his axiomatic declarations irrespective of (and possibly in contradiction to) what he measures in the real world; of course, if $P$ is not about the real world, then he has no other choice. Note that 'observer', 'measurement' and 'axiomatic declaration' are not part of the theory syntax in an NAFL theory. To see this, assume to the contrary that $Q$ is the formalized version of the proposition that "At $t=1$ the observer has measured (axiomatically declared) the cat to be alive". Then QM does not prove either $Q$ or $\neg Q$, but requires $Q \vee \neg Q$ to be a theorem, in violation of the Main Postulate of NAFL (see Proposition 1). It follows that $Q$ cannot be in the theory syntax of $\mathrm{QM}$; however, $Q \vee \neg Q$ is a valid deduction (and hence $Q$ is legitimate) in the proof syntax of QM. NAFL also correctly handles the temporal nature of truth via the time-dependence of $\mathrm{QM}^{*}$. If $P$ is observed at $t=1$, then the proposition $R$ that "The cat was alive for $0 \leq t<1$ " can be formalized in NAFL because $R$ applies only for $t \geq 1$; $R$ does not conflict with the superposed state $P \& \neg P$, which applies for $0<t<1$.

Let $A(B)$ be an undecidable proposition of QM about a given particle $\mathrm{X}$ (its distant entangled counterpart Y) such that QM classically proves the equivalence $A \Leftrightarrow B$. In NAFL, $A \Leftrightarrow B$ is deducible in the proof syntax of QM. If no measurements are made, the observer sets $\mathrm{QM}^{*}=\mathrm{QM}$ and both $A$ and $B$ are in the superposed state; note that $A \& \neg B$ (or $B \& \neg A$ ) is nonclassically true in the resulting model $\mathcal{M}$ of QM, which explains why $A \Leftrightarrow B$ cannot be a theorem of QM and is therefore not in its theory syntax (see Sec. 1). If $A$ is measured at a given time, then the observer sets $\mathrm{QM}^{*}=\mathrm{QM}+A$ and it follows that $\mathrm{QM}^{*}$ must prove $B$ in NAFL. Thus the axiomatic declaration of $A$ in $\mathrm{QM}^{*}$ entails the simultaneous axiomatic declaration of $B$ (with respect to QM) and there is no mystery associated with entanglement in NAFL. It is only when $A$ is interpreted strictly as a 'measurement' on the particle $\mathrm{X}$, as is necessary in conventional QM, that one is at a loss to explain how a simultaneous 'measurement', namely $B$, happens on its distant entangled counterpart Y. Non-locality is not a problem in NAFL, which rejects the relativity theories and non-Euclidean geometries [3] for essentially 
the same reason that it accepts superposition and entanglement - the Main Postulate.

\section{References}

[1] Radhakrishnan Srinivasan, "Quantum superposition justified in a new non-Aristotelian finitary logic", Pittsburgh Philosophy of Science Archive, Preprint Number 635, May 2002, http://philsci-archive.pitt.edu/archive/00000635/.

[2] Radhakrishnan Srinivasan, "Platonism in classical logic versus formalism in the proposed non-Aristotelian finitary logic", Pittsburgh Philosophy of Science Archive, Preprint Number 1166, May 2003, http://philsci-archive.pitt.edu/archive/00001166/.

[3] See the author's eprints in the Pittsburgh Philosophy of Science Archive: http://philsci-archive.pitt.edu/perl/user_eprints?username=sradhakr. See also the author's poster "Relativistic determinism: the clash with logic" at the International Conference on the Ontology of Spacetime, Concordia University, Montreal, May 11-14, 2004, http://alcor.concordia.ca/ scol/seminars/conference/abstracts.html/. 